\title{
Urticaria Pigmentosa de presentación atípica en la infancia: reporte de un caso clínico
}

\author{
Catherina Moll-Manzur ' ${ }^{1}$ Camila Downey 2, María Teresa Dossi C ${ }^{2}$
}

Resumen - La Urticaria Pigmentosa es el tipo más frecuente de mastocitosis cutánea (MC) de inicio en la edad pediátrica, representando el $75 \%$ de las MC. Se presenta con máculas y/o pápulas de color amarillo a café-rojizo, usualmente con signo de Darier positivo y prurito. El 88\% presenta compromiso de tronco. En este artículo compartimos el caso de Urticaria Pigmentosa de presentación atípica: predominio de lesiones en cara y sin compromiso de tronco.

Palabras clave: Urticaria Pigmentosa, mastocitosis.

Abstract - Urticaria Pigmentosa is the most common form of cutaneous mastocytosis (CM) starting in childhood and accounts for $75 \%$ of all the CM cases. It presents with yellow to brown-reddish macules or papules, usually with positive Darier sign and itching. Trunk involvement is present in $88 \%$ of patients. In this article we describe a case of Urticaria Pigmentosa with an atypical presentation: facial predominance without trunk involvement.

Keywords: Urticaria Pigmentosa, mastocytosis.

Fecha de envío: 25 de noviembre de 2015 - Fecha de aceptación: 31 de mayo de 2016

\section{Introducción}

Las mastocitosis son un grupo heterogéneo de enfermedades, caracterizadas por la acumulación de mastocitosis en la piel (mastocitosis cutáneas) y/o en órganos extracutáneos (mastocitosis sistémicas). La mayoría de las formas pediátricas corresponden a formas exclusivamente cutáneas. La Urticaria Pigmentosa (UP), también llamada mastocitosis cutánea máculo-papular, es la forma de mastocitosis pediátrica más frecuente y afecta predominantemente el tronco. A continuación, presentamos un caso clínico de UP de presentación atípica, con predominio de lesiones en cara y sin compromiso de tronco.

\section{Caso clínico}

Paciente de sexo femenino de 3 años de edad, sana, con historia de lesiones cutáneas persistentes, pruriginosas localizadas principalmente en cara y brazos, desde los 6 meses de edad. No presentaba dolor abdominal, osteoarticular ni cefalea. El examen físico evidenció la presencia de máculas de color café de diferentes tamaños, localizadas en mejillas (Figura 1) y en la cara extensora de las extremidades superiores, sin compromiso de tronco. El signo de Darier era positivo. Tenía dermografismo rojo, sin hepatoesplenomegalia ni adenopatías. El diagnóstico diferencial incluyó Urticaria Pigmentosa, histiocitosis cefálica benigna e hiperpigmentación posinflamatoria.

(1) Facultad de Medicina, Universidad de Chile. Santiago, Chile.

(2) Departamento de Dermatología, Facultad de Medicina, Pontificia Universidad Católica de Chile. Santiago, Chile.

Autor de correspondencia: mtdossi@gmail.com 


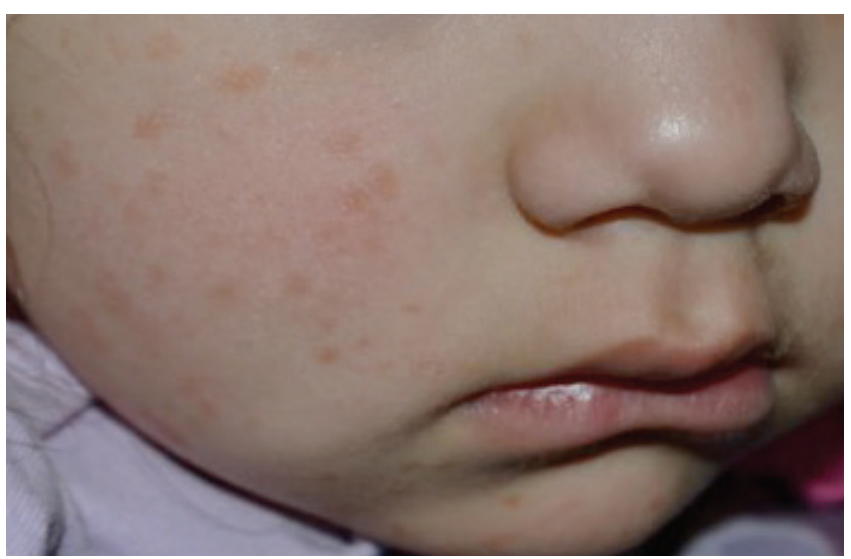

Figura 1: Apariencia clínica de la UP. Máculas de color café de diferentes tamaños, localizadas en mejillas, nariz y mentón.

Los exámenes de laboratorio revelaron: hemograma, velocidad de sedimentación y pruebas hepáticas normales, triptasa sérica: 1.33 ug/L (normal) y ecotomografía abdominal normal.

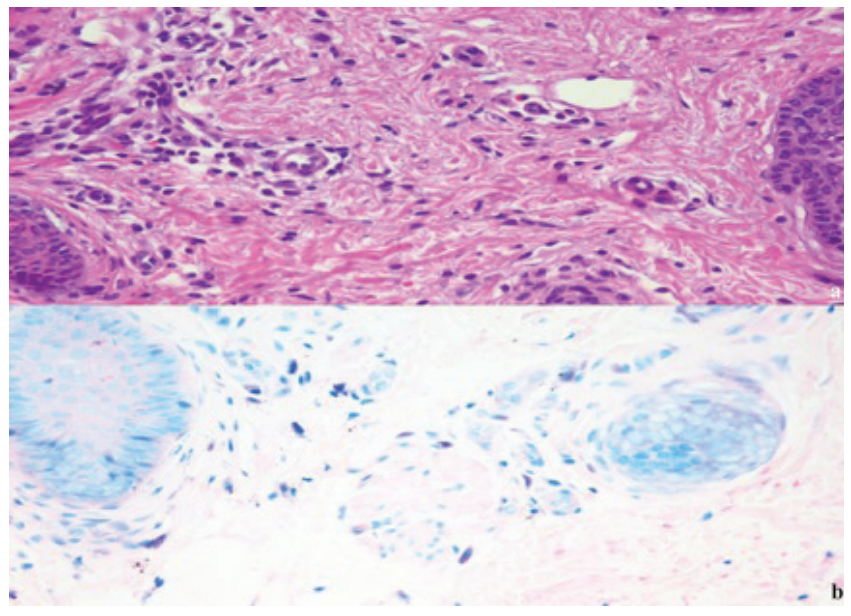

Figura 2: (a) Estudio histopatológico de la UP. Tinción de Hematoxicilina-eosina muestra dermis con infiltrado perivascular superficial linfocitario y de mastocitos abundantes.(b) Tinción de Giemsa en muestra histopatológica de la lesión.

Existe presencia de infiltrados mastocitarios.

El estudio histopatológico fue consistente con UP (Figuras 2a y 2b). La paciente se manejó con levocetirizina clorhidrato, con buena respuesta.

\section{Discusión}

La mayoría de las mastocitosis en la edad pediátrica corresponden a formas exclusivamente cutáneas y transitorias. Aproximadamente el $90 \%$ de las mastocitosis pediátricas se presenta en menores de 2 años, con una frecuencia hombre-mujer de 1.5: 1 (Ben-Amitai et al., 2005; Meni et al., 2015).

De las mastocitosis cutáneas (MC), el 75\% corresponde a la UP, seguida por el mastocitoma (20\%) y la mastocitosis cutánea difusa (5\%) (Meni et al., 2015).

La UP se presenta más frecuentemente con múltiples maculopápulas, de color café o rojizas, de forma oval y diferentes tamaños (variante polimórfica). La variante monomórfica es rara en niños y se presenta con lesiones homogéneas redondeadas, de menor tamaño que las de la variante polimórfica (Hartmann et al., 2016).

En cuanto a la distribución, las lesiones de UP se localizan de forma asimétrica y generalizada, comprometiendo el tronco, cabeza, cuello y extremidades (Hartmann et al., 2016). El compromiso de tronco se presenta en un $88 \%$ de los casos de la UP (Ben-Amitai et al., 2005).

El mastocitoma suele presentarse con una o un par de lesiones tipo placas o nódulos, de color amarillo-cafesoso o rojizo-cafesoso. La mastocitosis cutánea difusa, en cambio, se presenta con un eritema difuso, aumento de las marcas de la piel e infiltración con frecuentes vesículas.

El signo de Darier es altamente sensible y específico, encontrándose presente entre el 70\%-90\% de todos los casos de MC (Hannaford \& Rogers, 2001; Akoglu et al., 2006; Meni et al., 2015; Azaña et al., 2016b). Este se produce al rozar la lesión, produciéndose una urticación intensa. Puede asociarse además a prurito y dermografismo.

Un porcentaje importante se presenta con síntomas secundarios de la degranulación mastocitaria. El más frecuente es el flushing (24,5\% de los casos), seguido por los síntomas gastrointestinales, el dolor óseo y la cefalea. La anafilaxia se produce en un $10 \%$ de los niños con MC (Hartmann et al., 2016). 
Tabla 1: Factores que exacerban una degranulación mastocitaria (Modificado de (Hannaford \& Rogers, 2001; Azaña et al., 2016b, a).

\begin{tabular}{|c|c|c|}
\hline \multirow[t]{4}{*}{ 1) } & \multicolumn{2}{|l|}{ Agentes físicos } \\
\hline & . Calor & · Irritación mecánica de la piel \\
\hline & · Ejercicio & Procedimientos invasivos (quirúrgicoso \\
\hline & - Cambios bruscos de temperatura & endoscópicos del tracto gastrointestinal) \\
\hline \multirow[t]{2}{*}{ 2) } & \multicolumn{2}{|l|}{ Emocionales } \\
\hline & \multicolumn{2}{|l|}{ Ansiedad } \\
\hline \multirow[t]{7}{*}{ 3) } & \multicolumn{2}{|l|}{ Drogas } \\
\hline & - Antiinflamatorios (Aspirina y AINEs) & - Alcaloides (quinina, escopolamina) \\
\hline & - Anestésicos locales & - Medios de contraste iodados \\
\hline & - Antibióticos (anfotericina B, polimixina B, vancomicina) & - Narcóticos \\
\hline & - Antitusígenos & · Hidroxiurea \\
\hline & Opioides & - Alcohol \\
\hline & - Relajantes musculares e inductores & - Expansores plasmáticos \\
\hline \multirow[t]{3}{*}{ 4) } & \multicolumn{2}{|l|}{ Picaduras } \\
\hline & Abeja & Hormigas de fuego \\
\hline & Avispa & Serpientes \\
\hline \multirow[t]{5}{*}{ 5) } & \multicolumn{2}{|l|}{ Comidas } \\
\hline & - Colorantes & Chocolate \\
\hline & - Frutos secos & Carne ahumada \\
\hline & - Frutas cítricas & - Tomate \\
\hline & - Crustáceos & - Habas \\
\hline
\end{tabular}

Puede ocurrir una exacerbación sintomática ante ciertos factores, entre los que destacan factores físicos (calor y cambios bruscos de temperatura), emocionales (ansiedad), drogas (antiinflamatorios no esteroidales, anestésicos locales, opioides, narcóticos, relajantes musculares e inductores y algunos antibióticos), picaduras (heminópteros) y algunas comidas (frutos secos, crustáceos, chocolate) entre otros, que se detallan en la tabla 1 (Hannaford \& Rogers, 2001; Heide et al., 2008; Azaña et al., 2016a; Lange et al., 2016).

La severidad de los síntomas sistémicos no se ha correlacionado con un compromiso sistémico. Sin embargo, el hallazgo de organomegalia (hepatoesplenomegalia y linfoadenopatías) se ha considerado como un fuerte indicador de enfermedad sistémica (Carter et al., 2015).

Como estudio inicial a un paciente con mastocitosis pediátrica puede solicitarse un hemograma completo y niveles de triptasa sérica. La mayoría de los niños con UP presenta valores normales de triptasa sérica al diagnóstico. Si bien en adultos es clara la asociación entre niveles altos de TS y la infiltración de la médula ósea, esta asociación no está tan clara en pediatría (Hartmann et al., 2016). Niveles elevados de triptasa se han encontrado en niños con extenso compromiso cutáneo; los cuales tienen mayor riesgo de presentar síntomas potencialmente graves debido a la liberación de mediadores de los mastocitos (Azaña et al., 2016a).
El diagnóstico de las MC es clínico y se confirma con una biopsia de lesiones cutáneas (y de la piel circundante en caso de sospecharse una mastocitosis difusa cutánea) (Hannaford \& Rogers, 2001). En estos casos, la presencia infiltrados mastocitarios cutáneos confirma el diagnóstico.

El tratamiento de las $\mathrm{MC}$ es esencialmente sintomático. En general, no se requiere ninguna terapia, sin embargo, en niños con excesivo prurito y/o enrojecimiento deberán evitarse los factores que exacerben los síntomas (tabla 1).

La terapia sistémica en base a antihistamínicos $\mathrm{H}_{1}$ constituye la primera línea de tratamiento farmacológico y resulta efectiva en reducir el prurito (Zegpi-Trueba et al., 2015). Puede además combinarse con el uso de antihistamínicos $\mathrm{H}_{2}$ (Castells et al., 2011). El cromoglicato de sodio oral (15-20 mg/kg/día) y los antileucotrienos generalmente se usan cuando los síntomas no mejoran con antihistamínicos (Azaña et al., 2016b). En niños mayores de 2 años, pueden considerarse los corticoides tópicos por períodos de 3-6 semanas. La fototerapia con psoralenos combinados con UV-A o UV-B se reserva para casos raros de compromiso cutáneo masivo, con síntomas severos que no responden a las terapias antes mencionadas y/o con repetidas formaciones de ampollas (Hannaford \& Rogers, 2001; Azaña et al., 2016b). 
El pronóstico de la UP es generalmente bueno, con un curso benigno y autolimitado (Carter et al., 2015). La mayoría de los niños con UP presenta una remisión parcial o total de las lesiones en la pubertad (Hartmann et al., 2016).

Las UP son descritas en tronco en alrededor de un $90 \%$ de los casos pediátricos (Ben-Amitai et al., 2005). Este es un ejemplo con afectación exclusivamente facial y de extremidades superiores, por lo tanto, es una patología que debiera considerarse dentro del diagnóstico diferencial de lesiones que comprometen la cara en niños.

\section{Contribuciones y reconocimientos:}

Catherina Moll-Manzur: redacción del manuscrito. Camila Downey: revisión y aprobación del manuscrito. María Teresa Dossi C: revisión y aprobación del manuscrito. Ninguno de los autores presenta algún conflicto de interés.

\section{Referencias:}

Akoglu G, Erkin G, Cakir B, Boztepe G, Sahin S, Karaduman A, Atakan N, Akan T \& Kolemen F. (2006). Cutaneous mastocytosis: demographic aspects and clinical features of 55 patients. Journal of the European Academy of Dermatology and Venereology: JEADV 20, 969-973.

Azaña JM, Torrelo A \& Matito A. (2016a). Update on Mastocytosis (Part 1): Pathophysiology, Clinical Features, and Diagnosis. Actas dermo-sifiliograficas 107, 5-14.

Azaña JM, Torrelo A \& Matito A. (2016b). Update on Mastocytosis (Part 2): Categories, Prognosis, and Treatment. Actas dermo-sifiliograficas 107, 15-22.

Ben-Amitai D, Metzker A \& Cohen HA. (2005). Pediatric cutaneous mastocytosis: a review of 180 patients. The Israel Medical Association journal: IMAJ 7, 320-322.

Carter MC, Clayton ST, Komarow HD, Brittain EH, Scott LM, Cantave D, Gaskins DM, Maric I \& Metcalfe DD. (2015). Assessment of clinical findings, tryptase levels, and bone marrow histopathology in the management of pediatric mastocytosis. The Journal of allergy and clinical immunology 136, 1673-1679 e1671-1673.
Castells M, Metcalfe DD \& Escribano L. (2011). Diagnosis and treatment of cutaneous mastocytosis in children: practical recommendations. American journal of clinical dermatology 12, 259-270.

Hannaford R \& Rogers M. (2001). Presentation of cutaneous mastocytosis in 173 children. The Australasian journal of dermatology 42, 15-21.

Hartmann K, Escribano L, Grattan C, Brockow K, Carter MC, Alvarez-Twose I, Matito A, Broesby-Olsen S, Siebenhaar F, Lange M, Niedoszytko M, Castells M, Oude Elberink JN, Bonadonna P, Zanotti R, Hornick JL, Torrelo A, Grabbe J, Rabenhorst A, Nedoszytko B, Butterfield JH, Gotlib J, Reiter A, Radia D, Hermine O, Sotlar K, George TI, Kristensen TK, Kluin-Nelemans HC, Yavuz S, Hagglund $H$, Sperr WR, Schwartz LB, Triggiani M, Maurer M, Nilsson G, Horny HP, Arock M, Orfao A, Metcalfe DD, Akin C \& Valent P. (2016). Cutaneous manifestations in patients with mastocytosis: Consensus report of the European Competence Network on Mastocytosis; the American Academy of Allergy, Asthma \& Immunology; and the European Academy of Allergology and Clinical Immunology. The Journal of allergy and clinical immunology 137, 35-45.

Heide R, Beishuizen A, De Groot H, Den Hollander JC, Van Doormaal JJ, De Monchy JG, Pasmans SG, Van Gysel D, Oranje AP \& Dutch National Mastocytosis Work G. (2008). Mastocytosis in children: a protocol for management. Pediatric dermatology 25, 493-500.

Lange M, Lugowska-Umer H, Niedoszytko M, Wasag B, Limon J, Zawrocki A, Nedoszytko B, Sobjanek M, Plata-Nazar K \& Nowicki R. (2016). Diagnosis of Mastocytosis in Children and Adults in Daily Clinical Practice. Acta dermato-venereologica 96, 292-297.

Meni C, Bruneau J, Georgin-Lavialle S, Le Sache de Peufeilhoux L, Damaj G, Hadj-Rabia S, Fraitag S, Dubreuil P, Hermine O \& Bodemer C. (2015). Paediatric mastocytosis: a systematic review of 1747 cases. The British journal of dermatology 172, 642-651.

Zegpi-Trueba MS, Hasbun-Acuna P \& Berroeta-Mauriziano D. (2015). [Mastocytosis: Case report and literature review]. Revista chilena de pediatria. 